\title{
Relationship of backward walking to clinical outcome measures used to predict falls in the older population: A factor analysis
}

\author{
Carol A. Maritz ${ }^{1 *}$, Karin Grävare Silbernagel ${ }^{2}$ and Ryan Pohlig ${ }^{3}$
}

*Correspondence: c.maritz@usciences.edu

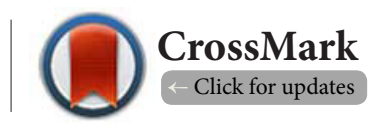

'Associate Dean and Professor of Physical Therapy, Samson College of Health Sciences, University of the Sciences, Philadelphia, PA 19104, USA.

${ }^{2}$ Assistant Professor, Department of Physical Therapy, University of Delaware, STAR Health Sciences Complex Campus, 540 South College Avenue Newark, DE 19713 USA.

${ }^{3}$ Sr. Biostatistician, Dean's Office, University of Delaware, STAR Health Sciences Complex Campus, 540 South College Avenue, Newark, DE 19713 USA.

\begin{abstract}
Background: Backward walking has been found to be a more sensitive measure to detect fallers than forward walking. It involves greater reliance on neuromuscular control to make up for the lack of vision. In fact, backward walking speed has been determined to more accurately identify fallers in the older population than forward walking. This study examined if backward walking measures more than one underlying factor while also examining the relationship between it and other clinical measures used to identify falls risk.

Methods: A convenience sample of 57 older adults (10 males and 47 females) with a mean age of 78.8 years (SD 8.9 years) participated in this cross-sectional study. Subjects walked backward on the GAITRite ${ }^{\circledR}$ computerized walkway system recording gait velocity, stride and step length, stance and swing time, single and double support. Additionally, subjects were tested on clinical measures commonly used to assess falls risk which included the Timed up and go test, Four square step test, Activity-specific balance confidence scale, single heel rise test and the 30-second chair stand test. An exploratory factor analysis with Maximum Likelihood extraction and Varimax rotation was performed on various aspects of backward walking. The relationship between the backwards walking factor scores and selected clinical measures was tested using Spearman correlations and regression.
\end{abstract}

Results: Results revealed that there are two factors associated with backward walking: cadence and velocity. Cadence was only mildly correlated to 30-second chair stand test, $r=0.29$; whereas velocity was highly correlated with each of the known predictors of falls used, all $r>0.44$.

Conclusion: Given theses findings, a clinician could quickly measure backward walking velocity and count the number of steps an individual takes in order to screen those at-risk for falls.

Keywords: Falls, gait, backward walking, older adults, balance, balance confidence

\section{Introduction}

Falls are the number one cause of injury, fractures, and death among the older population [1]. In fact, one third of adults over the age of 60 will experience one or more falls annually. There are older adults who are so fearful of falling they stop being physically active whether or not they have actually fallen [2].
It is widely known that this activity limitation is largely due to a loss of confidence in their ability to perform common functional activities [3].

A recent study proposed the use of backward walking as a clinical measure of mobility in the older population [4]. Compared to younger adults, older adults have demonstrated 
Maritz et al. Physical Therapy and Rehabilitation 2017,

http://www.hoajonline.com/journals/pdf/2055-2386-4-14.pdf

doi: $10.7243 / 2055-2386-4-14$

decreased velocity and stride length, and increased gait variability during both forward and backward walking $[4,5]$. However, according to Fritz et al, backward walking speed has been found to more accurately identify elderly fallers than forwarding walking speed [4]. In fact, all the fallers in this study by Fritz et al had a backward walking speed of less than $0.6 \mathrm{~m} / \mathrm{s}$ [4].

Although the cause of falls is multifactorial, lower extremity weakness and decreased balance are two significant factors associated with falls in the older population $[2,6,7]$. Older adults tend to fall while performing mobility tasks such as moving from sit to stand or walking especially when the individual needs to move backwards in order to navigate around tight spaces [8]. Backward stepping and walking require a greater reliance on neuromuscular control, proprioception, and protective reflexes because of the lack of visual cues [9]. This could be one of the reasons for backward walking being a more sensitive measure in detecting fallers compared to forward walking $[4,5]$.

When evaluating gait performance several spatiotemporal gait measures are used such as gait speed, cadence, stance and swing time, and time in double versus single support. Since gait is considered multidimensional, it is of interest to evaluate the underlying constructs of walking ability. A previous study has reported that forward walking consists of three components that accounted for $87 \%$ of the variability in spatiotemporal parameters [10]. These components were considered to measure pace, rhythm and variability. Since backward walking has been found to be more sensitive to detect fallers $[\mathbf{4 , 5}]$, it is of interest to evaluate if backward walking has similar constructs or if there are additional factors that explains the variability in performance.

To our knowledge, there are no studies that have examined the underlying constructs of backward walking and the relationship between backward walking and other measures relating to falls such as lower extremity and calf muscle strength, balance, and walking. Therefore, the aims of this study were 1) to evaluate if backward walking measures more than one underlying factor and 2) to determine the relationship between backward walking and other clinical measures used to identify falls risk such as the Timed up and go, Four square step test, Activity-specific balance confidence scale, Single heel rise test and the 30-second chair stand test. We hypothesized that backward walking would consist of multiple factors and that these factors would be connected to the clinical falls outcome measures.

\section{Materials and methodology Subjects}

This cross-sectional study included a sample of convenience of 57 older adults (10 males and 47 females). The mean age of the participants was 78.8 years (SD 8.9 years). Mean height was calculated at $161 \mathrm{~cm}$ (SD 9.3) and mean body weight was $73.1 \mathrm{~kg}$ (SD 18.1). Participants were recruited from two senior centers and one local church. All participants were community dwelling older adults, and were able to ambulate with or without an assistive device and demonstrated an ability to follow multi-step directions. The Institutional Review Board of University of the Sciences approved this study. All participants were consented prior to participation.

As researchers, we were interested in evaluating the relationship between BW and commonly used clinical measures that we believe are directly related to BW. In the literature it is described that falls risk is related to lower extremity strength, balance, general mobility and fear [11-13]. For this study, we chose one standard clinical test as representative of each domain for evaluating the relationship with BW. The participants were therefore tested on the following outcome measures along with the BW task: Timed up and go (TUG) test for mobility, Four Square Step test (FSST) for dynamic balance, Activity-Specific Balance Confidence Scale (ABC) for balance confidence, the Single-leg Heel Rise test for calf muscle strength and 30-second Chair Stand test for lower extremity strength.

\section{Backward Walking}

ProtoKinetics Movement Analysis Software, PKMAS, (ProtoKinetics, LLC, Havertown, PA) was used to collect footfall data and compute spatiotemporal gait measures using an instrumented walkway, GAITRite ${ }^{\oplus}$ Mat(CIR Systems, Franklin, $\mathrm{NJ}$ ). The walkway consists of a $61 \times 366 \mathrm{~cm}$ carpet embedded with pressure sensors. Spatiotemporal gait measures collected on GAITRite ${ }^{\circledR}$ walkways have been found to be valid and reliable in the elderly [14-16].

Participants walked forward at a comfortable pace for 3 trials (down and back) on the 12-foot computerized walkway in order to familiarize themselves with the carpet. Then they walked backward on the walkway for 1 trial, which included no fewer than 10 strides. Hollman et al found that data collected from 10-20 strides on the GAITRite ${ }^{\oplus}$ was found to be a reliable method for measuring velocity and cadence [17]. All participants were instructed to walk 2 meters before and after the walkway to allow for acceleration and deceleration. All trials were completed without an assistive device while wearing a gait belt and guarded by one member from the research team. The variables analyzed during the backward walking gait cycle included: gait speed $(\mathrm{m} / \mathrm{s})$, cadence (steps/ $\mathrm{min})$, average step and stride length $(\mathrm{cm})$, stance and swing time (sec), single support (\% of the gait cycle), double support (\% of the gait cycle).

\section{Timed Up and Go test (TUG)}

The Timed Up and Go test (TUG) is a standardized outcome measure used to assess mobility in older adults [18]. The test involves timing participants while they stand from being seated in a chair, stand up, walk 3 meters, turn around, walk back 3 meters and sit down. This test was performed 3 times after which the average of the three trials (in seconds) was 
calculated and used for analysis. Community-dwelling older adults should be able to perform the TUG in less than $12 \mathrm{sec}-$ onds [19]. According to Bohannon [20] individuals between the ages of 70-79 years, the mean age of this study's participants, should be able to perform the TUG in 9.2 seconds. The TUG was originally developed as a clinical measure to assess balance in the elderly and it has shown to have excellent inter and intra-rater reliability. The intra-class correlation coefficient (ICC) has been reported to be $>0.95$ [8].

\section{The Four Square Step Test (FSST)}

Four Square Step test is a measure of dynamic balance that assesses the person's ability to step over objects forward, sideways, and backwards. Patient steps over four one inch pieces of PVC pipe set-up like a cross on the floor [21].

Community-dwelling older adults should be able to perform the FSST in less than 18 seconds [19]. This test has shown to have excellent inter -rater and test-retest reliability. The intraclass correlation coefficient (ICC) have been reported to be 0.99 and 0.98 respectively [21].

\section{The Activity Balance Confidence Scale (ABCS)}

The Activities-Specific Balance Confidence Scale (ABC Scale) is a 16-item self-report survey assessing the confidence a patient has in their balance during certain activities [22]. The patient rates their confidence on a scale of $0 \%$ (no confidence) to $100 \%$ (complete confidence) for each item. Age, as well as education level is factored into evaluating the data from the survey. The less confidence a patient has, the lower their scores will be on the survey, and they will have a higher risk for falls. The results also show that a higher percentage is correlated to being a more mobile, active, and functional person. This tool has excellent internal consistency with a Cronbach alpha of 0.95 [23].

\section{Single-leg Heel Rise test}

The muscular endurance test is a standing heel-rise test that has previously been evaluated for reliability [24-26]. The MuscleLab $^{\circledast}$ (Ergotest Technology) measurement system was used for the evaluation. A linear encoder, that uses a spring-loaded string connected to a sensor inside the unit is used with the string attached to the heel. When the string is pulled the sensor outputs a series of digital pulses that are proportional to the distance travelled. The heel-rise test is performed on one leg at a time with the participant standing on a box with an incline of $10^{\circ}$. For balance the participants were allowed to place the hand, at shoulder height, against the wall. The participant was instructed to go as high as possible on each heel-rise and was asked to perform as many heelrises as possible. The test was terminated when the patient stopped, or could not perform a proper heel-rise. The height of the heel-rise (the distance moved superiorly by the heel in reference to the box) had to be a minimum of $2 \mathrm{~cm}$ to be counted as one repetition. The numbers of heel-rises as well as the height of each heel-rise were collected, and the total work (the body weight $x$ total distance) in joules was used for data analysis. The mean of the left and right side heel-rise work was used for data analysis. This test has been shown to have good reliability (ICC 0.78-0.84) [25,27].

\section{The 30-Second Chair Stand Test (30-sCST)}

This test provides insight into a person's lower body strength and endurance [28]. It links strength findings to the ability to perform everyday tasks such as climbing stairs, getting in and out of a vehicle or a bathtub [29]. To perform this test, subjects were seated in a chair without arm rests. The height of the seat was 17 inches from the floor. They were asked to stand up and sit down as many times as they could in 30 seconds. The number of times that they could stand up was then recorded. This test has been shown to have excellent reliability with an ICC of 0.84 for males and 0.92 for females [28].

\section{Data analysis}

Descriptive data are reported as mean, standard deviation (SD) and range (minimum and maximum). An Exploratory Factor Analysis (EFA) was performed using Maximum Likelihood extraction and Varimax rotation on various aspects of backwards walking: including: velocity, cadence, stance time, swing time, single support, double support, average stride length and average step length. To make sure the correct number of factors were extracted visual analysis of a scree plot was used in conjunction with a parallel analysis and fit indices were examined. Lastly, to make sure the factors that were extracted have real world meaning factor scores were found and then correlated with commonly used clinical outcome measures including heel-rise work, TUG, 30-second CST, ABC, and FSST test. Alpha of .05 was used for all analyses.

\section{Results}

The mean, standard deviation and range of scores on the clinical outcome measures are presented in Tables 1 and 2

Table 1. Results on the clinical outcome measures.

\begin{tabular}{lll}
\hline Outcomemeasure & $\begin{array}{l}\text { Mean (SD), } \\
\mathbf{n = 5 7}\end{array}$ & $\begin{array}{l}\text { Range } \\
\text { (min-max) }\end{array}$ \\
\hline TUG(sec) & $11.51(3.73)$ & $6-24.09$ \\
FSST (sec) & $13.42(4.72)$ & $7.55-32.58$ \\
ABC (\%) & $78.17(14.85)$ & $35-99.38$ \\
30-sCST (reps) & $11.17(3.47)$ & $2-23$ \\
Right heelrise reps & $17.9(13.6)$, & $0-55$ \\
Left heel-rise reps & $18.5(13.4)$ & $0-69$ \\
Combined heel-rise reps & $18.2(12.5)$ & $0-60$ \\
Right heel-rise work (joule) & $700.3(583.6)$ & $0-2190$ \\
Left heel-rise work (joule) & $762.4(693.2)$ & $0-2637$ \\
Combined heel-rise work (joule) & $731.3(599.3)$ & $0-2413$ \\
\hline
\end{tabular}

TUG: Timed Up and Go test; FFST Four Square Step test; ABC: the Activity-Specific Balance Confidence Scale; 30-sCST: The 30-second Chair Stand test 
Maritz et al. Physical Therapy and Rehabilitation 2017,

http://www.hoajonline.com/journals/pdf/2055-2386-4-14.pdf

doi: $10.7243 / 2055-2386-4-14$

Table 2. Results from the backward walking using GAITRite mat and PKMAS software.

\begin{tabular}{lll}
\hline Backward walking variables & $\begin{array}{l}\text { Mean (SD) } \\
\mathbf{n = 5 8}\end{array}$ & Range (min-max) \\
\hline Velocity (sec) & $46.6(19.87)$ & $8.45-89.99$ \\
Cadence (steps/min) & $101.82(16.81)$ & $66.16-154.94$ \\
Stance time (sec) & $0.88(0.16)$ & $0.55-1.42$ \\
Swing time (sec) & $0.33(0.06)$ & $0.21-0.05$ \\
Double support (\%) & $44.64(7.84)$ & $31.72-61.31$ \\
Single support (\%) & $27.7(3.91)$ & $19.38-34.22$ \\
Average stride length $(\mathrm{cm})$ & $54.8(21)$ & $11.7-95$ \\
Average step length $(\mathrm{cm})$ & $27.3(10.5)$ & $5.8-47.5$ \\
\hline
\end{tabular}

SD: Standard deviation

includes the mean, standard deviation and range of scores of the backward walking gait variables.

\section{Exploratory Factor Analysis}

An EFA was performed on measures of backwards walking: velocity, cadence, stance time, swing time, single support, double support, average stride length and average step length. All assumptions were tested: no missing data, multicollinearity, linearity, multivariate normality, no outliers, and factorability. There was multi-collinearity among the eight initial measures, double and single support were almost perfectly correlated, $r=-0.99$, as were stride and step length, $r=0.99$, lastly velocity was highly correlated with stride and step length, both $r=0.94$. Due to this, stride and step length were removed as well as single support. Secondly, due to multivariate non-normality, as tested by Mardia's tests of Skewness and Kurtosis, the EFA was performed on the Spearman Correlation Matrix. To find the appropriate number of factors, a Principal Component Analysis (PCA) with scree plot, Parallel Analysis were performed, and fit indices were examined. The PCA suggested, examining the scree plot, and considering Eigen Values greater than one suggested that two factors be extracted. The two factors extracted accounted for $94.5 \%$ of the variance, the parallel analysis resulted in the same conclusion [30]. The Kaiser-MeyerOlkin value was 0.62 (above the recommend .5) and Bartlett's test was significant, $p<0.001$. Lastly, the factor correlation matrix was examined to see if an oblique rotation would be needed, but factors were only weakly correlated $r=0.20$.

Maximum Likelihood extraction with a Varimax rotation was used [31]. The first factor included cadence, stance time, and swing time. The second factor included velocity, double support, swing time and stance time. Both swing and stance time were 'complex' measures, loading on both factors (see Factor Loadings in Table 3). Factor scores were calculated using the Regression Method after standardizing the variables.

\section{Relationship with Outcomes}

To make sure the factors were meaningful in a real world setting, the factor scores were correlated with the five outcomes:
Table 3. Factor Loadings.

\begin{tabular}{lll}
\hline Variable & Factor 1 & Factor 2 \\
\hline Cadence & -0.983 & -- \\
Stance Time & 0.874 & -0.456 \\
Swing Time & 0.728 & 0.583 \\
Double Support & -- & 0.990 \\
Velocity & -- & 0.820 \\
\hline
\end{tabular}

heel-rise work, TUG, 30-sec chair stand test, ABC, and FSST. Factor 1 was not correlated with any of the outcomes (all $\mathrm{p}>0.05$ ), whereas Factor 2 was significantly correlated with all outcomes (all $p<0.05$ ), (Table 4). To make the results more meaningful for clinicians a representative measure from each factor was chosen to illustrate meaning, cadence for the first factor and velocity for the second.

Additionally, regression analyses were used to predict each of the outcomes using both cadence and velocity. Velocity was a significant predictor for each outcome after adjusting for cadence (Table 5), identical results were obtained when factor scores were used.

\section{Discussion}

Falls in older adults often occur when they are moving backwards [8]. As a result, we felt that this study that examines the underlying constructs of backward walking and the relationship between them would provide valuable information for physical therapists. The factor analysis revealed that there were 2 factors associated with backward walking: 1) cadence and 2) velocity. The first factor included cadence, stance time, and swing time while the second factor included velocity, double support, swing time and stance time. To determine if the factors had clinical meaning, we alsoexamined the relationship between the factors and commonly used clinical outcome measures that included TUG, 30-second Chair Stand test, FSST, ABC Scale, and Heel Rise Work.

Cadence was only mildly correlated with the 30-second Chair Stand test, a gross measure of lower extremity strength and endurance. This suggests that there may be other explanations for increased cadence among older adults during BW. Laufer [5] found that when older adults attempt to increase their velocity during backward walking, they are only able to increase cadence not stride length. While possible explanations for this might include lack of hip and trunk extension range of motion as well as age-related declines in calf muscle strength, further study is needed to explain these findings [32].

Backward walking velocity was highly correlated [33] with the selected clinical measures suggesting that components of each measure are included in the act of backward walking. These components included lower extremity strength, neuromuscular control (proprioception, vestibular system, and reaction time) and balance confidence. An individual's fear of falling and balance confidence might have a significant impact on BW velocity since they lack the visual cues they 
Maritz et al. Physical Therapy and Rehabilitation 2017,

http://www.hoajonline.com/journals/pdf/2055-2386-4-14.pdf

Table 4. Correlation between clinical outcomes and the factors.

\begin{tabular}{lllllllll}
\hline Spearman's rho & Factor $\mathbf{1}$ & $\mathbf{p}$ & Factor $\mathbf{2}$ & $\mathbf{p}$ & Cadence & $\mathbf{p}$ & Velocity & $\mathbf{P}$ \\
\hline HR_work & -.10 & 0.44 & .45 & $<.001$ & .17 & .212 & .44 & .001 \\
TUG & .13 & 0.32 & -.72 & $<.001$ & -.23 & .081 & -.76 & $<.001$ \\
30-sCST & -.21 & 0.11 & .61 & $<.001$ & .29 & .028 & .60 & $<.001$ \\
ABC & -.08 & 0.57 & .60 & $<.001$ & .17 & .208 & .60 & $<.001$ \\
FSST & .01 & 0.92 & -.65 & $<.001$ & -.12 & .367 & -.64 & $<.001$ \\
\hline
\end{tabular}

HR work: Heel rise work; TUG: Timed Up and Go test; 30-sCST: The 30-second Chair Stand test;

ABC: Activity-Specific Balance Confidence Scale; FFST Four Square Step test

Table 5. Results from regression analyses to predict outcome.

\begin{tabular}{llllll}
\hline & & $\mathbf{b}$ & Std. Error & $\mathbf{t}$ & Sig. \\
\hline HR work & Velocity & 14.25 & 3.99 & 3.58 & .001 \\
\multirow{3}{*}{ TUG } & Cadence & -2.42 & 4.71 & -0.51 & .61 \\
& Velocity & -.14 & .02 & -6.85 & $<.001$ \\
\multirow{2}{*}{ Chair Stand Test } & Celocity & .09 & .02 & 4.20 & $<.001$ \\
& Cadence & .01 & .03 & 0.36 & .718 \\
ABC & Velocity & .44 & .09 & 4.80 & $<.001$ \\
& Cadence & -.09 & .11 & -0.80 & .428 \\
\multirow{2}{*}{ FSST } & Velocity & -.16 & .03 & -5.97 & $<.001$ \\
& Cadence & .05 & .03 & 1.58 & .119 \\
\hline
\end{tabular}

HR work: Heel rise work; TUG: Timed Up and Go test; 30-sCST:

The 30-second Chair Stand test;ABC: Activity-Specific Balance

Confidence Scale; FFST Four Square Step test

rely on during forward walking. The absence of vision during backward walking places higher demands on already age impaired sensorimotor function [34]. This can result in the older adult taking shorter strides and using a slower velocity as they try to minimize the feeling of falling $[4,35,36]$. Other explanations for decreased backward walking velocity include lower extremity muscle weakness, hip flexion contractures and generalized deconditioning all of which are common in the older population.

Verghese et al performed a factor analysis of forward walking and also found that velocity and cadence were related to two different factors. In their study, only the cadence factor (termed rhythm factor) was associated with global cognitive decline. In our study, we found that only the velocity factor was correlated with our clinical outcome measures but none of our outcomes related to cognitive ability. Further research is needed to determine if in backward walking the cadence factor is also related to cognitive ability or other clinical measures not evaluated in this study.

Our results agree with previous studies that have examined spatiotemporal changes, specifically velocity, that during $\mathrm{BW}$ in the older population velocity is significantly reduced as compared to younger individuals $[4,5]$. Additionally, we found that backward walking velocity was highly correlated [33] with known predictors of falling-TUG, FSST, ABC, and HRT suggesting that $B W$ velocity could be used to identify those at risk for falls. Based on this information, a clinician could quickly measure backward walking velocity and count the number of steps an individual takes in order to help screen those who may be at-risk for falls. The findings from this screening would then substantiate a more in depth assessment using some of the above-mentioned clinical measures.

This study had a few limitations. The cross-sectional design only allows for the assessment of the variables over a given period of time. Although the sample size was somewhat small it was diverse in age, ethnicity and activity level leading to greater generalizability. Future studies should explore the use of backward walking as an intervention to see if it would have a beneficial effect on balance, strength, mobility and balance confidence.

\section{Conclusion}

The results of this study support that there are convincing relationships between backward walking velocity and commonly used clinical outcome measures to assess falls risk in the older population. Based on these findings, a busy clini- 
Maritz et al. Physical Therapy and Rehabilitation 2017,

http://www.hoajonline.com/journals/pdf/2055-2386-4-14.pdf

doi: $10.7243 / 2055-2386-4-14$

cian may only need to assess BW velocity to screen those at risk for falls.

\section{List of abbreviations \\ BW: Backward walking \\ TUG: Timed up and go \\ FSST: Four square step test \\ $A B C$ : Activity-specific balance confidence \\ EPA: Exploratory factor analysis \\ PCA: Principal component analysis}

\section{Competing interests}

The authors declare that they have no competing interests.

Authors' contributions

\begin{tabular}{|l|c|c|c|}
\hline Authors' contributions & CAM & KSG & RP \\
\hline Research concept and design & $\checkmark$ & $\checkmark$ & -- \\
\hline Collection and/or assembly of data & $\checkmark$ & $\checkmark$ & $\checkmark$ \\
\hline Data analysis and interpretation & $\checkmark$ & $\checkmark$ & $\checkmark$ \\
\hline Writing the article & $\checkmark$ & $\checkmark$ & $\checkmark$ \\
\hline Critical revision of the article & $\checkmark$ & $\checkmark$ & $\checkmark$ \\
\hline Final approval of article & $\checkmark$ & $\checkmark$ & $\checkmark$ \\
\hline Statistical analysis & -- & -- & $\checkmark$ \\
\hline
\end{tabular}

\section{Acknowledgement}

The authors would like to thank the participants from the Jewish Community Center.

Publication history

Editor: Gordon John Alderink, Grand Valley State University, USA. Received: 29-Jun-2017 Final Revised: 13-Sep-2017

Accepted: 25-Oct-2017 Published: 14-Nov-2017

\section{References}

1. Important Facts about Falls | Home and Recreational Safety | CDC Injury Center, (n.d.). 2016. | Website

2. Murphy SL, Williams CS and Gill TM. Characteristics associated with fear of falling and activity restriction in community-living older persons. $J$ Am Geriatr Soc. 2002; 50:516-20. I Article I PubMed Abstract | PubMed FullText

3. Friedman SM, Munoz B, West SK, Rubin GS and Fried LP. Falls and fear of falling: which comes first? A longitudinal prediction model suggests strategies for primary and secondary prevention. J Am Geriatr Soc. 2002; 50:1329-35. | Article | PubMed

4. Fritz NE, Worstell AM, Kloos AD, Siles AB, White SE and Kegelmeyer DA. Backward walking measures are sensitive to age-related changes in mobility and balance. Gait Posture. 2013; 37:593-7. | Article | PubMed

5. Laufer Y. Effect of age on characteristics of forward and backward gait at preferred and accelerated walking speed. J Gerontol A Biol Sci Med Sci. 2005; 60:627-32. | PubMed

6. Cronin NJ, Barrett RS, Lichtwark G, Mills PM and Carty CP. Decreased lower limb muscle recruitment contributes to the inability of older adults to recover with a single step following a forward loss of balance. J Electromyogr Kinesiol. 2013; 23:1139-44. | Article I PubMed

7. Melzer I, Krasovsky T, Oddsson LI and Liebermann DG. Age-related differences in lower-limb force-time relation during the push-off in rapid voluntary stepping. Clin Biomech (Bristol, Avon). 2010; 25:989-94. | Article | PubMed

8. Schoene D, Wu SM, Mikolaizak AS, Menant JC, Smith ST, Delbaere K and Lord SR. Discriminative ability and predictive validity of the timed up and go test in identifying older people who fall: systematic review and meta-analysis. J Am Geriatr Soc. 2013; 61:202-8. | Article I PubMed

9. Yang YR, Yen JG, Wang RY, Yen LL and Lieu FK. Gait outcomes after additional backward walking training in patients with stroke: a randomized controlled trial. Clin Rehabil. 2005; 19:264-73. | Article | PubMed

10. Verghese J, Wang C, Lipton RB, Holtzer R and Xue X. Quantitative gait dysfunction and risk of cognitive decline and dementia. J Neurol Neurosurg Psychiatry. 2007; 78:929-35. | Article | PubMed Abstract | PubMed FullText

11. Rubenstein LZ. Falls in older people: epidemiology, risk factors and strategies for prevention. Age Ageing. 2006; 35 Suppl 2:ii37-ii41. | Article I PubMed

12. Ambrose AF, Paul G and Hausdorff JM. Risk factors for falls among older adults: a review of the literature. Maturitas. 2013; 75:51-61. | Article | PubMed

13. Moreland JD, Richardson JA, Goldsmith $\mathrm{CH}$ and Clase CM. Muscle weakness and falls in older adults: a systematic review and metaanalysis. J Am Geriatr Soc. 2004; 52:1121-9. | Article I PubMed

14. Bilney B, Morris $M$ and Webster K. Concurrent related validity of the GAITRite walkway system for quantification of the spatial and temporal parameters of gait. Gait Posture. 2003; 17:68-74. I Article I PubMed

15. H.B. Menz, M.D. Latt, A. Tiedemann, M.M.S. Kwan and S.R. Lord. Reliability of the GAITRite ${ }^{\circledR}$ walkway system for the quantification of temporo-spatial parameters of gait in young and older people. Gait Posture. 2004; 20:20-25. | Article

16. Egerton T, Thingstad $\mathrm{P}$ and Helbostad JL. Comparison of programs for determining temporal-spatial gait variables from instrumented walkway data: PKmas versus GAITRite. BMC Res Notes. 2014; 7:542. Article | PubMed Abstract | PubMed FullText

17. Hollman JH, Childs KB, McNeil ML, Mueller AC, Quilter CM and Youdas JW. Number of strides required for reliable measurements of pace, rhythm and variability parameters of gait during normal and dual task walking in older individuals. Gait Posture. 2010; 32:23-8. | Article | PubMed

18. Podsiadlo D and Richardson S. The timed "Up \& Go": a test of basic functional mobility for frail elderly persons. J Am Geriatr Soc. 1991; 39:142-8. | Article | PubMed

19. Bischoff HA, Stahelin HB, Monsch AU, Iversen MD, Weyh A, von Dechend M, Akos R, Conzelmann M, Dick W and Theiler R. Identifying a cut-off point for normal mobility: a comparison of the timed 'up and go' test in community-dwelling and institutionalised elderly women. Age Ageing. 2003; 32:315-20. | PubMed

20. Bohannon RW. Reference values for the timed up and go test: a descriptive meta-analysis. J Geriatr Phys Ther. 2006; 29:64-8. | Article | PubMed

21. Dite $W$ and Temple VA. A clinical test of stepping and change of direction to identify multiple falling older adults. Arch Phys Med Rehabil. 2002; 83:1566-71. | Article | PubMed

22. Myers AM, Fletcher PC, Myers AH and Sherk W. Discriminative and evaluative properties of the activities-specific balance confidence (ABC) scale. J Gerontol A Biol Sci Med Sci. 1998; 53:M287-94. I PubMed

23. Talley KM, Wyman JF and Gross CR. Psychometric properties of the activities-specific balance confidence scale and the survey of activities and fear of falling in older women. J Am Geriatr Soc. 2008; 56:328-33. Article | PubMed

24. Silbernagel KG, Nilsson-Helander K, Thomee R, Eriksson BI and Karlsson $J$. A new measurement of heel-rise endurance with the ability to detect functional deficits in patients with Achilles tendon rupture. Knee Surg Sports Traumatol Arthrosc. 2010; 18:258-64. | Article I PubMed

25. Moller M, Lind K, Styf J and Karlsson J. The reliability of isokinetic testing of the ankle joint and a heel-raise test for endurance. Knee Surg Sports Traumatol Arthrosc. 2005; 13:60-71. | Article | PubMed

26. G. Svantesson. Ulla; Osterberg, Ulrika; Thomee, Roland; Grimby, Muscle fatigue in a standing heel-rise test. Scand. J. Rehabil. Med. 1998; 30:6772. | Article

27. Svantesson U, Carlsson U, Takahashi H, Thomee R and Grimby G. 
Comparison of muscle and tendon stiffness, jumping ability, muscle strength and fatigue in the plantar flexors. Scand J Med Sci Sports. 1998; 8:252-6. | Article | PubMed

28. Jones CJ, Rikli RE and Beam WC. A 30-s chair-stand test as a measure of lower body strength in community-residing older adults. Res $Q$ Exerc Sport. 1999; 70:113-9. | Article | PubMed

29. Chandler JM, Duncan PW, Kochersberger G and Studenski S. Is lower extremity strength gain associated with improvement in physical performance and disability in frail, community-dwelling elders? Arch Phys Med Rehabil. 1998; 79:24-30. | Pdf | PubMed

30. Szczepaniak K, Astel A, Simeonov V, Tsakovski S, Biziuk M, Bode P and Przyjazny A. Comparison of dry and living Sphagnum palustre moss samples in determining their biocumulative capability as biomonitoring tools. J Environ Sci Health A Tox Hazard Subst Environ Eng. 2007; 42:1101-15. | Article | PubMed

31. B.G. Tabachnick and L.S. Fidell. Using multivariate statistics (6th ed.). 2012.

32. Kerrigan DC, Todd MK, Della Croce U, Lipsitz LA and Collins JJ. Biomechanical gait alterations independent of speed in the healthy elderly: evidence for specific limiting impairments. Arch Phys Med Rehabil. 1998; 79:317-22. | Pdf | PubMed

33. Mukaka MM. Statistics corner: A guide to appropriate use of correlation coefficient in medical research. Malawi Med J. 2012; 24:69-71. | PubMed Abstract | PubMed FullText

34. Callisaya ML, Blizzard L, McGinley JL, Schmidt MD and Srikanth VK. Sensorimotor factors affecting gait variability in older people--a population-based study. J Gerontol A Biol Sci Med Sci. 2010; 65:386-92. | Article | PubMed

35. Chamberlin ME, Fulwider BD, Sanders SL and Medeiros JM. Does fear of falling influence spatial and temporal gait parameters in elderly persons beyond changes associated with normal aging? J Gerontol A Biol Sci Med Sci. 2005; 60:1163-7. I PubMed

36. Laufer Y. Age- and gender-related changes in the temporal-spatial characteristics of forwards and backwards gaits. Physiother Res Int. 2003; 8:131-42. | Article | PubMed

\section{Citation:}

Maritz CA, Silbernagel KG and Pohlig R. Relationship of backward walking to clinical outcome measures used to predict falls in the older population: A factor analysis.

Phys Ther Rehabil. 2017; 4:14.

http://dx.doi.org/10.7243/2055-2386-4-14 